\title{
Tai Chi in Parkinson's Disease: A Preliminary Randomized, Controlled, and Rater-Blinded Study
}

\author{
Roger Kurlan $^{1 *}$, Roye Evans², Sandra Wrigley¹, Shannon McPartland1, Rami Bustami ${ }^{3}$, \\ Ann Cotter ${ }^{2}$ \\ ${ }^{1}$ Atlantic Neuroscience Institute, Overlook Medical Center, Summit, NJ, USA \\ ${ }^{2}$ Integrative Medicine, Atlantic Health System, Morristown, NJ, USA \\ ${ }^{3}$ Biostatistics, Atlantic Health System, Morristown, NJ, USA \\ Email: 'roger.kurlan@atlantichealth.org
}

Received 3 December 2014; accepted 17 December 2014; published 13 January 2015

Copyright (C) 2015 by authors and Scientific Research Publishing Inc.

This work is licensed under the Creative Commons Attribution International License (CC BY).

http://creativecommons.org/licenses/by/4.0/

c) (i) Open Access

\begin{abstract}
Background: Given the limited benefits of current treatments for Parkinson's disease (PD), interventions that might provide supplementary benefits would be of value. The traditional Chinese medicine practice of Tai Chi has been said to improve some aspects of PD, particularly imbalance. Methods: Preliminary randomized, controlled, and rater-blinded clinical trial of Tai Chi, focusing on its effects on global motor, daily function, mood and quality of life. Subjects continued their standard medical therapy and were randomly assigned to Tai Chi (16 weekly classes, expert trainer, practice at home between classes) or no Tai Chi (control group) in a 2:1 ratio. The primary outcome measure was the total motor score of the Unified Parkinson's Disease Rating Scale (UPDRS) and this was scored by an experienced rater who was blinded to the treatment assignment. The same rater scored the Schwab and England Activities of Daily Living Scale. The patient-completed Geriatric Depression Scale, PD (quality of life) Questionnaire-39, and fall diary were also analyzed. Results: 44 subjects participated with 29 assigned to Tai Chi and 15 serving as controls. Tai Chi was well-tolerated. Seven subjects withdrew prior to completion ( 2 from Tai Chi, 5 from control). We found no significant difference between treatment groups in the change in scores from baseline to end of intervention for any of the scales. Trends toward a benefit of Tai Chi were observed for individual UPDRS items (depression, finger tapping, hand movements, posture). Conclusions: Tai Chi does not appear to improve global measures in patients with PD. The practice may have benefits for PD, but these appear to be largely restricted to specific motor tasks and perhaps mood rather than being a global functional response. More study is needed to clarify and establish efficacy.
\end{abstract}

\footnotetext{
${ }^{*}$ Corresponding author.
}

How to cite this paper: Kurlan, R., Evans, R., Wrigley, S., McPartland, S., Bustami, R. and Cotter, A. (2015) Tai Chi in Parkinson's Disease: A Preliminary Randomized, Controlled, and Rater-Blinded Study. Advances in Parkinson's Disease, 4, 9-12. http://dx.doi.org/10.4236/apd.2015.41002 


\section{Keywords}

\section{Parkinson's Disease, Tai Chi, Mind-Body, Treatment}

\section{Introduction}

Currently available therapies for Parkinson's disease (PD) temporarily improve symptoms, but the illness results in progressive disability. Given the progressive nature of the disease and the limited time window during which current treatments are effective, there has been increasing interest in identifying interventions that can supplement existing therapies to improve motor control and function. It has been shown, for example, that physical therapy and exercise can improve aspects of gait and balance [1]. Mind-body therapies focus on the relationships among brain, mind, body and spirit and a number have been applied to neurological disorders [2]. Tai Chi is a traditional Chinese medicine technique that incorporates body movement, breathing and attentional training to improve medical symptoms and maintain health. The practice of Tai Chi involves slow body positions that flow from one to the next continuously and promotes improved posture, balance, flexibility, relaxation, well-being and mental concentration [3]. Tai Chi is considered appropriate for patients with PD given its emphasis on slow movements of the body.

Despite widespread use of Tai Chi by patients with PD and anecdotal reports of its benefits, only a few studies have been published. Li et al. studied 17 patients with mild to moderate PD and found that following a 5 day, 90 minutes per day program of Tai Chi there were measurable improvements in speed of movement and functional reach [4]. Hackney and Earhart studied 33 PD patients who were randomized to Tai Chi (20 one hour-long training sessions within a 13 week period) or no Tai Chi (control) and found that the Tai Chi group improved more than the control group on measures of PD severity, gait and balance [5]. Li et al. studied 195 patients with PD who participated in Tai Chi for 60 minutes twice weekly for 24 weeks [6]. Evidence of improvement in some measured aspects of postural stability was reported. In order to obtain more standard and systematic information about the potential efficacy and tolerability of Tai Chi for patients with PD, particularly focusing on more global motor, functional, mood and quality of life outcomes, we carried out a randomized, controlled, examiner-blinded preliminary clinical trial.

\section{Materials and Methods}

Following institution-approved informed consent, a total of 44 subjects were enrolled into the trial. Enrollment criteria included age 18 or older, being diagnosed with idiopathic PD by a movement disorders specialist (RK), and being ambulatory (use of an assistive device such as cane or walker was allowed). Subjects were excluded if they had previous experience with Tai Chi or if they had cognitive or other motor impairment (e.g., pain, arthritis) that would interfere with their ability to properly participate in the Tai Chi activities. Subjects were randomized in a 2:1 ratio (in order to facilitate enrollment) to Tai Chi or no Tai Chi (control group). Subjects in both groups continued their best medical therapy (no subject had neurosurgical treatment). Subjects assigned to Tai Chi attended 16 weekly group therapy classes led by a qualified and experienced instructor. Each class lasted 60 minutes; caregivers were encouraged to participate in the classes and to assist their partner with PD. Each Tai Chi class involved the following protocol: quiet sitting with breathing and visualizations (7 minutes), gentle stretching of neck, back and legs (5 minutes), gentle whole body self massage (5 minutes), seated or standing brocades (two palms press heaven, single palm press heaven, bow and arrow, cow looks over shoulder to gaze at moon, punch with angry eyes, dancing with the rainbow; 15 minutes), standing balance forms (walking on thin ice, dragon stepping, pushing the swing; 15 minutes), and clearing the body (3 minutes). Subjects were encouraged to practice Tai Chi at home between classes.

Each subject underwent a baseline assessment prior to randomization which included the Unified PD Rating Scale (Parts I and II; UPDRS) [7] and Schwab and England Activities of Daily Living Scale (S \& E) [8], both scored by an experienced rater who remained blinded to treatment assignment throughout the tria, the self-reported Geriatric Depression Scale (GDS) [9], and the disease-specific quality of life scale the PD Questionnaire-39 (PDQ-39) [10]. Patients were instructed on how to complete a standardized self-report diary of any fall that took place throughout the duration of the study. The same assessments were repeated within 10 days after 
completing the 16 week trial. The same examiner was used for each subject at both the baseline and final evaluations and these raters were blinded to treatment assignment (patients and family members were forbidden from revealing treatment). Fall diaries were collected monthly. The study was powered to detect a difference between the treatment groups of at least a 5 point change in the total motor score of the UPDRS, considered a minimally clinically important difference [11]. The Tai Chi and control groups were compared in terms of change (baseline to follow-up) in outcomes using the t test or the Mann-Whitney U test. Corrections for multiple comparisons were included when assessing individual items in the UPDRS. Subjects who dropped out during the study were excluded from the analyses.

\section{Results}

A total of 44 subjects enrolled in the trial. There were 27 men and 17 women. Average age was 72 years (range 44 to 90 years). Average duration of PD was 4.2 years (range 6 weeks to 18 years). Twenty-nine subjects were assigned to Tai Chi and 15 subjects were assigned to the control group. None of the subjects in the control group reported engaging in Tai Chi or any new exercise activity. Seven subjects withdrew before completing the study ( 2 in the Tai Chi group, 5 controls). Thirty-two subjects reported no falls on their diaries so their diary data were not analyzed. There were no statistically significant differences between the treatment groups for changes from baseline to post-treatment for the total motor UPDRS, S \& E, GDS, and PDQ-39 scores or on the fall diary. As summarized in Table 1, trends toward improvement following Tai Chi were identified for some individual items of the UPDRS (depression, finger tapping, hand movements, posture). No similar trends were identified in the control group.

\section{Discussion}

Our randomized, controlled, blinded rater assessment of a 16 week course of Tai Chi did not identify any statistically significant evidence of benefit for measured overall PD severity (UPDRS), quality of life, severity of depression or fall frequency. Our results differ from those of Hackney and Earhart who identified improvement on the UPDRS after Tai Chi [5]. Our study differed in that Tai Chi training occurred weekly rather than twice each week and by having live, rather than videotape-based assessments of the UPDRS. It is possible that a class frequency greater than once weekly is needed to achieve more substantial improvement, although our subjects were asked to practice Tai Chi at home between classes. Alternatively, our live interviews and examinations may have provided more accurate measures of outcome. Another published study of Tai Chi in PD did not include the UPDRS measure [4] and only involved 5 days of intervention. The final published study utilized mechanical, quantitative measures of postural stability which we did not employ.

The three published studies of Tai Chi in PD identified improvements in specific motor tasks, including 50 foot speed walk [5], six-minute walk [4], backward walking [4], tandem stance test [4], timed up and go [4] [5], functional reach [4]-[6], Berg Balance Scale [4], maximum excursion [6], directional control [6], and stride length [6] which may have been more sensitive to change than the more global measures we employed. We were specifically interested if Tai Chi might produce global motor and functional benefits beyond specific motor tasks, yet this response was not evident. It is possible that our sample size may have been too small to detect less than major effects of Tai Chi. We did identify trends suggesting that the Tai Chi group may have improved for some of the individual UPDRS motor items, including finger tapping, hand movements and posture. We also found a trend toward improvement of the UPDRS depression item, but there was no significant change on the GDS. Of interest, a recently published randomized controlled trial found that use of Tai Chi augmented the benefits of

Table 1. UPDRS Items with a trend toward improvement following Tai Chi.

\begin{tabular}{|c|c|c|c|}
\hline & \multicolumn{3}{|c|}{ Group } \\
\hline Factor (Mean \pm SD) & Control (N = 15\%, 34\%) & Tai Chi (N = 29\%, 66\%) & p-value ${ }^{*}$ \\
\hline UPDRS I (depression) & $0.3 \pm 0.5$ & $-0.1 \pm 0.6$ & 0.054 \\
\hline UPDRS III (Finger tapping) & $0.4 \pm 0.7$ & $-0.07 \pm 0.9$ & 0.090 \\
\hline UPDRS III (Posture) & $0.1 \pm 0.3$ & $-0.3 \pm 0.7$ & 0.093 \\
\hline UPDRS III (Rapid altern. movement of hands) & $2.0 \pm 0.9$ & $-0.3 \pm 0.7$ & 0.16 \\
\hline
\end{tabular}


the antidepressant drug escitalopram in the treatment of geriatric depression [12]. Our study was not restricted to patients with depression or to a geriatric population. The trends were of small effect sizes and may not be of clinical significance. The lower drop-out rate in our Tai Chi group may, however, support the presence of some clinical benefit.

\section{Conclusion}

Overall, our study suggests that Tai Chi is well tolerated by patients with PD but no clear changes in measures of overall PD motor severity, daily function, quality of life or mood are identified. Our results suggest that Tai Chi may lead to clinical improvement, but any benefit appears to be largely restricted to specific motor tasks and perhaps mood, rather than being a global motor or functional response. More study is needed with larger sample sizes to clarify and establish this efficacy.

\section{Acknowledgements}

This study was supported by the Grotta Fund for Senior Care of the Jewish Community fund of Metro West New Jersey, Whippany, NJ.

\section{Author Disclosure Statement}

No competing financial interests exist.

\section{References}

[1] Goodwin, V.A., Richards, S.H., Taylor, R.S., Taylor, A.H. and Campbell, J.L. (2008) The Effectiveness of Exercise Interventions for People with Parkinson's Disease: A Systematic Review and Meta-Analysis. Movement Disorders, 23, 631-640. http://dx.doi.org/10.1002/mds.21922

[2] Wahbeh, H., Elsas, E.-M. and Oken, B.S. 2008 () Mind-Body Interventions: Applications in Neurology. Neurology, 70, 2321-2328. http://dx.doi.org/10.1212/01.wnl.0000314667.16386.5e

[3] Li, F., Harmer, P., McAuley, E., et al. (2001) Tai Chi, Self-Efficacy, and Physical Function in the Elderly. Prevention Science, 2, 229-239. http://dx.doi.org/10.1023/A:1013614200329

[4] Li, F., Harmer, P., Fisher, K.J., et al. (2007) Tai Chi-Based Exercise for Older Adults with Parkinson's Disease: A Pilot-Program Evaluation. Journal of Aging and Physical Activity, 15, 139-151.

[5] Hackney, M.E. and Earhart, G.M. (2008) Tai Chi Improves Balance and Mobility in Patients with Parkinson’s Disease. Gait \& Posture, 28, 456-460. http://dx.doi.org/10.1016/j.gaitpost.2008.02.005

[6] Li, F., Harmer, P., Fitzgerald, K., et al. (2012) Tai Chi and Postural Stability in Patients with Parkinson's Disease. New England Journal of Medicine, 366, 511-519. http://dx.doi.org/10.1056/NEJMoa1107911

[7] Fahn, S. and Elton, R.L., Members of the UPDRS Development Committee (1987) Unified Parkinson's Disease Rating Scale. In: Fahn, S., Marsden, C.D., Goldstein, M. and Calne, C.D., Eds., Recent Developments in Parkinson’s Disease, Volume II, Florham Park, 153-163.

[8] Schwab, R.S. and England, A.C. (1969) Projection Technique for Evaluating Surgery in Parkinson's Disease. In: Gillingham, F.J. and Donaldson, I.M.L., Eds., Third Symposium on Parkinson's Disease, Edingurgh, Livingstone, 152157.

[9] Sheikh, J.I. and Yesavage, J.A. (1986) Geriatric Depression Scale (GDS): Recent Evidence and Development of a Shorter Version. Journal of Mental Health and Aging, 5, 165-173.

[10] Peto, V., Jenkinson, C. and Fitzpatrick, R. (1998) PDQ-39: A Review of the Development, Validation and Application of a Parkinson's Disease Quality of life Questionnaire and Its Associated Measures. Journal of Neurology, 245, S10S14. http://dx.doi.org/10.1007/PL00007730

[11] Schrag, A., Sampaio, C., Counsell, N., et al. (2006) Minimal Clinically Important Change on the Unified Parkinson's Disease Rating Scale. Movement Disorders, 21, 1200-1207. http://dx.doi.org/10.1002/mds.20914

[12] Lavretsky, H., Alstein, L.L., Olmstead, R.E., et al. (2011) Complementary Use of Tai Chi Augments Escitalopram Treatment of Geriatric Depression: A Randomized Controlled Trial. The American Journal of Geriatric Psychiatry, 19, 839-850. http://dx.doi.org/10.1097/JGP.0b013e31820ee9ef 
Scientific Research Publishing (SCIRP) is one of the largest Open Access journal publishers. It is currently publishing more than 200 open access, online, peer-reviewed journals covering a wide range of academic disciplines. SCIRP serves the worldwide academic communities and contributes to the progress and application of science with its publication.

Other selected journals from SCIRP are listed as below. Submit your manuscript to us via either submit@scirp.org or Online Submission Portal.
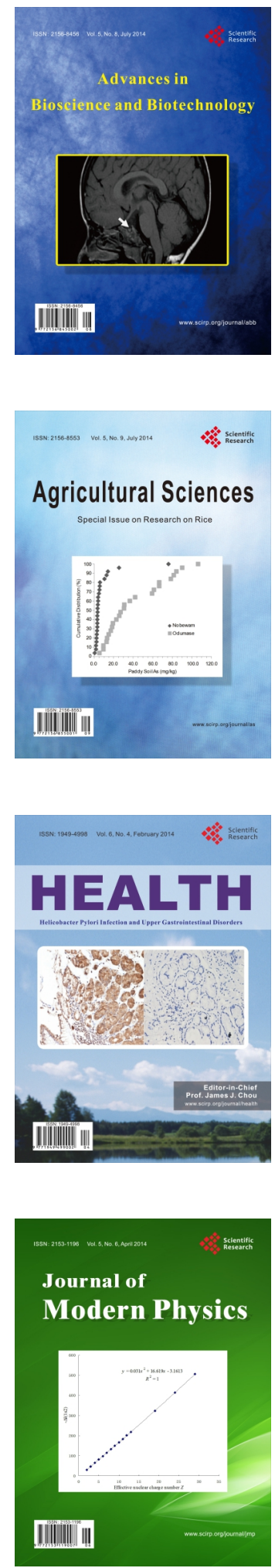
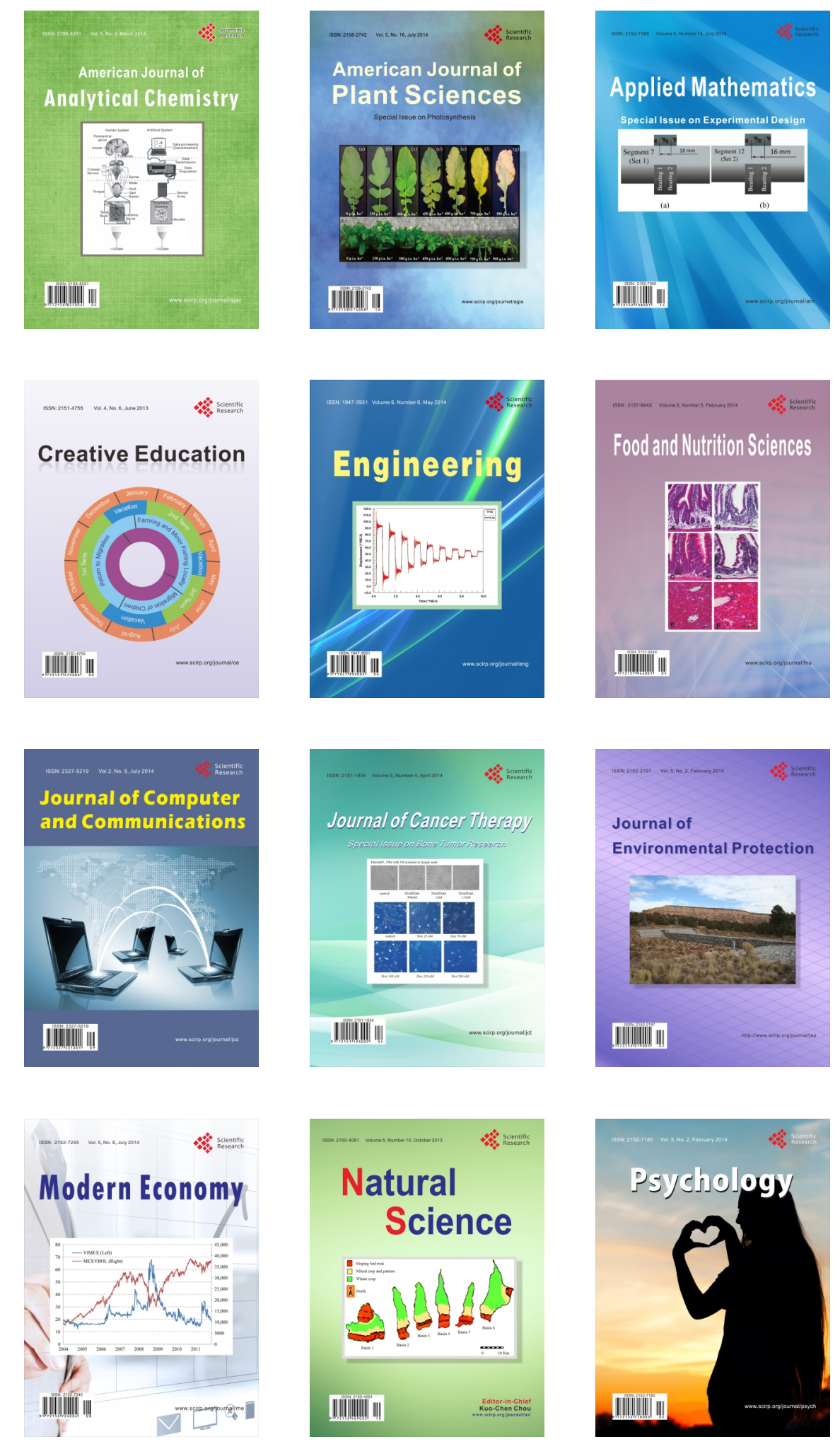